\title{
Planificación De Entrenamiento Deportivo Orientada Al Desarrollo De Los Fundamentos Técnicos Del Baloncesto Caso: Estudiantes Categoría Intermedia Distrito Chambo - Riobamba
}

\author{
Karolina Janeth Cando Brito \\ Magíster en Cultura Física y Entrenamiento Deportivo \\ Docente Unidad Educativa Riobamba \\ Henry Rodolfo Gutierrez Cayo \\ Magister en Entrenamiento Deportivo \\ Docente Universidad Nacional de Chimborazo \\ Hernán Leopoldo Ponce Bravo Phd. \\ Doctor en Actividad Física y Salud \\ Docente Universidad Nacional de Chimborazo \\ Christian Hernán Ponce Franco \\ Master en Rendimiento Deportivo y Salud \\ Alumno - Doctor en Actividad Física y Salud Universidad de Granada \\ Orlando David Mazón Moreno \\ Magister en Cultura Física y Entrenamiento Deportivo \\ Docente de la Escuela Superior Politécnica de Chimborazo
}

doi: 10.19044/esj.2017.v13n27p94 URL:http://dx.doi.org/10.19044/esj.2017.v13n27p94

\begin{abstract}
The present research work responds to the planning of sports training oriented towards the development of the technical fundamentals of basketball. Specifically, the study was applied in the Chambo-Riobamba district of Chimborazo, Ecuador. The study was conducted based on a previous study on the analysis of the technical fundamentals of basketball. It details the need to develop a plan that is oriented towards the students of the intermediate category of the district. Through student survey, this study aims to avert the problem that demonstrates the absence of an oriented planning that is reflected in the low sport performance in this sport. The methodology used was through direct observation. The surveys were conducted for students, coaches, and field research. As a result, it is possible to define and apply daily training plans to determine how the universe of this study was responding to the exercise plan. The plan is made up of 29 sessions divided into 1 macrocycle, 7 mesocycles, and 25 microcycles. In conclusion, we
\end{abstract}


ascertain that the sports training plan is a necessary tool for the physical, psychological, and skill preparation of the athletes in the practice of the technical fundamentals of the basketball. In addition, it demonstrated better results in the district and provincial competences of the Educational Units belonging to the objective of this study.

Keywords: Training plan, technical fundamentals, basketball, daily plan

\section{Resumen}

El presente trabajo de investigación responde a la planificación de entrenamiento deportivo orientada al desarrollo de los fundamentos técnicos del baloncesto, específicamente el estudio se lo aplicó en el distrito Chambo - Riobamba de Chimborazo, Ecuador. Se fundamentó en el estudio previo sobre el análisis a los fundamentos técnicos del baloncesto, en él se detalla la necesidad de elaborar una planificación orientada a los estudiantes de la categoría intermedia del distrito además, se demuestra la problemática al no existir una planificación orientada que se refleja en el bajo rendimiento deportivo en este deporte, por parte de los alumnos encuestados. La metodología empleada fue a través de la observación directa, las encuestas aplicadas a estudiantes y entrenadores y la investigación de campo, así se logra definir y aplicar los planes diarios de entrenamiento, que sirvió para comprobar como el universo de estudio fue respondiendo al plan de ejercicio. El plan está conformado por 29 sesiones repartidas en 1 macrociclo, 7 mesociclos y 25 microciclos. Como conclusión se obtuvo que el plan de entrenamiento deportivo es una herramienta necesaria para la preparación física, psicológica y de destreza para los deportistas en la práctica de los fundamentos técnicos del baloncesto y se demostró mejores resultados en las competencias distritales y provinciales de las Unidades Educativas pertenecientes al objeto de estudio.

Palabras clave: Plan de entrenamiento, fundamentos técnicos, baloncesto, plan diario

\section{Introducción}

La presente propuesta de planificación deriva del estudio realizado en base a los resultados del "Análisis de los fundamentos básicos técnicos del baloncesto en el rendimiento deportivo de los estudiantes categoría intermedia del circuito 06D01C02 distrito Chambo -Riobamba", mediante la cual se determinó que los fundamentos básicos técnicos del baloncesto en la categoría intermedia son; el dribling, el tiro y los pases mismos que se ponen de manifiesto durante gran parte del juego y que se deberían trabajar con mayor énfasis en el desarrollo y perfeccionamiento de estos. 
Además después de haber realizado los análisis de las encuestas aplicadas a jugadores y entrenadores por separado (cada cual con su respectivo instrumento de investigación), se llegó a la conclusión de que los estudiantes del área en estudio, presentaban un nivel de rendimiento deportivo considerablemente bajo, esto debido a las deficiencias técnicas observadas y al poco trabajo que se le dedica para el desarrollo de los fundamentos técnicos (Cando K., Guitierrez H., Ponce H., Aldas H. \& Mazón O., 2017).

\section{Antecedentes}

Una vez expuestos los resultados de las encuestas se demostró la necesidad de implantar una alternativa innovadora que vaya a cumplir con las expectativas de los estudiantes deportistas en cuanto a mejorar los fundamentos básicos técnicos del baloncesto, pues la sociedad actual requiere de entrenadores, monitores que sigan un proceso de manera tecnificada, que interactúan aprendiendo el uno del otro, además debemos manifestar que el baloncesto dentro de las instituciones educativas del circuito 06D01C02 no han presentado ninguna planificación, en consecuencia es importante motivar a los estudiantes de esta disciplina deportiva a través de una nueva forma de desarrollar las habilidades básicas con balón, como también acrecentar conocimientos nuevos, a través de la práctica deportiva y técnica.

Para la ejecución de la propuesta se realizaron previas investigaciones en el circuito 06D01C02 en las que se encuentran vinculadas las siguientes Instituciones: Unidad Educativa Riobamba, Unidad Educativa Camilo Gallegos, Instituto de música Vicente Anda Aguirre, en la que el Sr. Director Distrital Ing. Dimas Gaibor a raíz de que se inició esta investigación ha creído conveniente realizar un entrenamiento sistemático y progresivo en busca de fortalecer la práctica de baloncesto en cada uno de los estudiantes.

\section{Justificación}

La razón por la que se realiza el plan de entrenamiento y su aplicación en el rendimiento deportivo dirigido a deportistas, entrenadores y monitores es para contribuir al mejoramiento de los fundamentos básicos del baloncesto de los estudiantes deportistas del circuito, es para que los deportistas tengan una idea más clara sobre su sesión de entrenamiento personal, logrando de esta forma motivarlos y apoyarlos en cada una de sus clases.

Al enfocar este problema se debe tomar en cuenta la importancia que tiene éste trabajo investigativo en el distrito educativo, considerando que el tema es de relevancia porque está afectando al rendimiento deportivo al no contar con un plan estructurado para el desarrollo correcto de los 
fundamentos técnicos del baloncesto, y a la vez que se refleja en resultados negativos por parte de las instituciones investigadas. Cabe mencionar que se dio soporte y charlas a entrenadores y monitores los cuales estuvieron involucrados en esta actividad que fue en beneficio de los deportistas con el fin de mejorar su rendimiento.

Es por esta razón que se justificó la elaboración de un plan de entrenamiento utilizando elementos básicos para el desarrollo de los estudiantes los cuales fuero de mucha importancia para aquellos que integran el circuito.

\section{Beneficiarios}

Internos: Entrenadores, estudiantes deportistas del circuito educativo 06D01C02 del distrito Chambo - Riobamba de la provincia de Chimborazo.

\section{Ubicación:}

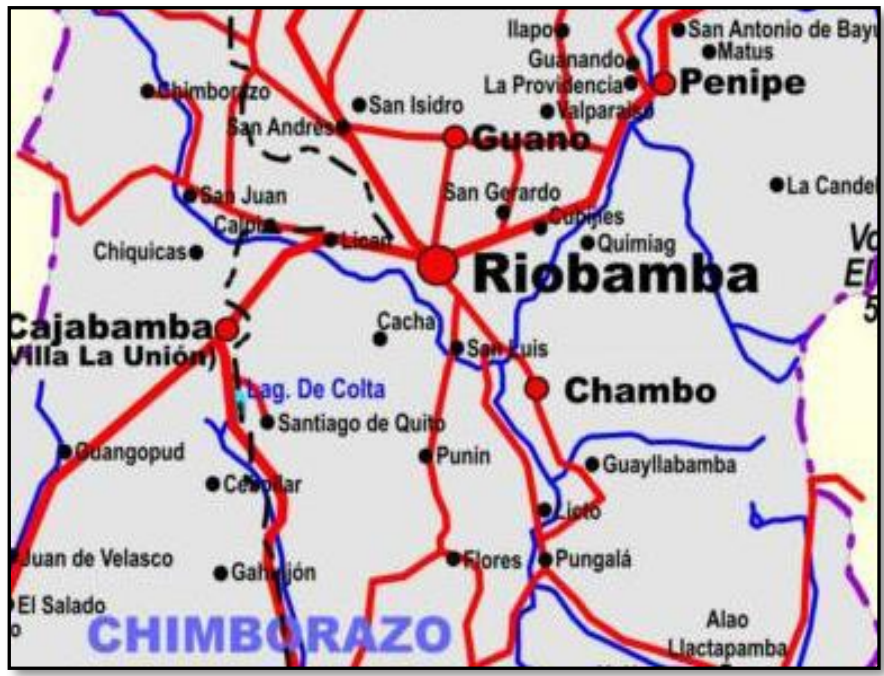

Figura 1. Ubicación Geográfica, circuito 06D01C02

\section{Tiempo estimado para la ejecución}

Inicio: Enero del 2015

Final: Julio del 2015

\section{Equipo Técnico Responsable}

La propuesta fue ejecutada por el equipo investigador en base a un desarrollo bibliográfico, documental y con los indicadores de la investigación previamente levantada, se enfocaron los objetivos dentro de lo que va a ser la creación del Plan de Entrenamiento, donde se buscó estrategias y técnicas adecuadas para el buen desarrollo de la Propuesta. 


\section{Objetivo General}

Desarrollar los fundamentos básicos técnicos del baloncesto mediante la aplicación de una planificación estructurada en porcentajes para una correcta asimilación de la técnica en categorías intermedias con los estudiantes deportistas del circuito 06D01C02.

\section{Objetivos Específicos}

a) Continuar la educación y desarrollo de habilidades y/o capacidades motrices básicas, atendiendo el lógico proceso evolutivo (Psico-fisiológico) característico en las edades de $14-15$ años de ambos sexos.

b) Consolidar la enseñanza de los elementos técnicos, vinculándolos a las acciones tácticas, así como en las concepciones básicas del juego, que logren el aumento de la capacidad resolutiva del alumno.

c) Confeccionar un Plan de entrenamiento en correspondencia con los principios básicos técnicos del baloncesto.

\section{Análisis de Factibilidad}

Después del análisis correspondiente del problema, se consideró que es plenamente factible la realización y culminación de la presente propuesta, por las siguientes razones:

Socio Cultural: Desde distintos ámbitos, permitan la ejercitación de una práctica pedagógica crítica y significativa, a partir de la construcción del conocimiento y el análisis reflexivo acerca de los fines y desafíos del nuevo siglo.

Tecnológica: La humanidad y el mundo progresa por eso el entrenamiento de los estudiantes deportistas del circuito debe estar manejada por personas altamente capacitadas y con un sistema de entrenamiento planificado, acorde a utilizar las tecnologías para reforzar el progreso competitivo que es la base fundamental de la sociedad en la actualidad.

Organizacional: La organización tanto Institucional como la parte metodológica es la base fundamental para que los estudiantes progresen con buenas bases en esta disciplina deportiva.

Equidad de Género: Debemos darnos cuenta que en las instituciones educativas ya no solo existe un solo sexo, ahora el Ministerio de Educación del Ecuador estableció que las Instituciones Educativas deben estar conformadas tanto hombres como mujeres, demostrando que todos somos iguales y cualquiera de los géneros son capaces de desarrollar fundamentos básicos del baloncesto.

Legal: Fundamentados en la LOEI (Ley organiza de educación intercultural), ley del deporte, en las cuales proponen y permiten las actividades deportivas en las instituciones educativas. 
Político: Se refiere a que el sistema o proyecto propuesto debe respetar los acuerdos, convenios y reglamentos internos de tipo empresarial, industrial, sindical, religioso, partidista, cultural, deportivo u algún otro relacionado con el ámbito del proyecto.

\section{Fundamentación Científica}

\section{Plan de Entrenamiento}

Planificar es anticipar proveer una secuencia lógica y coherente del desarrollo de las tareas que nos llegan alcanzar objetivos previamente definidos. Es un proceso que el entrenador sigue para seguir las líneas de orientación a corto o largo plazo. La planificación debe ser coordinada, orientada y evaluada (Jiménez, 2011).

\section{Planificación Deportiva}

La planificación es una propuesta teórica construida por la descripción, previsión, organización y diseño de los acontecimientos del entrenamiento, en una determinada fase de la vida deportiva de un deportista, así como los mecanismos de control que permitan modificar esos acontecimientos a fin de obtener un proceso de entrenamiento ajustado para lograr los resultados deseados en la competición (Jiménez, 2011).

\section{Periodización}

La periodización consiste en dividir el plan anual en fases de entrenamiento más cortas y manejables. Esta división mejora la organización del entrenamiento y permite a los entrenadores dirigir el programa sistemáticamente. La periodización se elabora en dependencia del deporte que se vaya a entrenar y del calendario de competiciones. La división del plan anual en ciclos más pequeños facilita la labor del entrenador, pudiendo tener un control más exhaustivo de la carga de entrenamiento (Torrijo, 2004).

\section{Fase de Preparación}

En cada uno de estos macros de entrenamiento se encuentra esta temporada donde el deportista sumara un buen trabajo aeróbico de forma gradual y segura, en los entrenamientos se debe de considerar un trabajo con intensidad baja o moderada el principal objetivo mejorar la capacidad aeróbica, resistencia ganar fuerza muscular e incrementar la flexibilidad (Mestre, 2013). 


\section{Fase de Construcción}

También llamado de especificidad, donde la intensidad de los entrenamientos aumentara, uno de los objetivos aumentar la capacidad para rendir al máximo en la distancia o deporte que se vaya a competir.

\section{Fase de Competición}

Este es el momento de indicar lo que se ha mejorado que tan exactos somos durante las fases anteriores, está marcada por la disminución en el volumen e intensidad para evitar llegar fatigados a las competencias.

\section{Fase de Transición o Recuperación}

La última fase de un buen plan de entrenamiento, cuyo objetivo será darle a nuestro cuerpo el tiempo necesario para descansar y recuperarse, luego de fases intensivas de entrenamiento.

\section{Componentes de la Planificación Deportiva}

De acuerdo a Alvarez (2004) y los autores Carrasco (2014), el proceso de entrenamiento se estructura en periodos temporales bien definidos:

$\checkmark$ Macrociclos (ciclos anuales: preparatorio, precompetitivo, competitivo y transición).

$\checkmark \quad$ Mesociclos (ciclos de 2 a 8 semanas).

$\checkmark \quad$ Microciclos (ciclos de aproximadamente una semana).

$\checkmark \quad$ Unidades de entrenamiento (cada sesión en particular).

\section{Macrociclo}

Es un periodo de entrenamiento planificado a largo plazo ya sea de 3 a 12 meses, además de ello incluye una fase competitiva.

\section{Particularidades}

$\checkmark \quad$ Tiene los contenidos y medios de entrenamiento en un periodo de tiempo.

$\checkmark \quad$ Respeta las dinámicas de la carga en cuanto a su carácter individual y especial como una unidad independiente.

\section{Mesociclo}

Conformado por una duración media puede ser de 3 a 6 semanas ( 3 a 6 de su preparación, es un proceso necesario y completo que produce un trabajo. 


\section{Particularidades}

$\checkmark \quad$ Acumulación: aumenta el potencial motor del deportista.

$\checkmark$ Transformación: concentración alta de carga de entrenamiento especializada.

$\checkmark \quad$ Realización: competición.

\section{Tipos de Mesociclo}

Acumulación: El objetivo principal elevar la preparación física y técnica, para sentar las bases para el desarrollo específico. Su duración al inicio de la preparación es más larga y en la medida que avanza el proceso de entrenamiento anual, se hace más corta.

Transformación: El objetivo principal trasformar el potencial motor obtenido en el mesociclo de acumulación, mediante un trabajo dirigido a la tolerancia a la fatiga y a la estabilidad de la técnica. Es un proceso intermedio entre el mesociclo de Acumulación y el de Realización.

Realización: Crea la predisposición psicológica para las competencias cercanas. Estos mesociclos son cortos y van en aumento en la medida que se acercan las competencias más importantes (Carrasco Dimas, Carrasco David \& Carrasco Darío, 2014).

\section{Microciclo}

Es la dosificación más concreta y operativa de los temas de entrenamiento y estos determinan la dinámica de la carga de trabajo como el efecto acumulativo del entrenamiento.

\section{Particularidades}

$\begin{array}{ll}\checkmark & \text { Ajuste } \\ \checkmark & \text { Carga } \\ \checkmark & \text { Impacto } \\ \checkmark & \text { Activación } \\ \checkmark & \text { Competición } \\ \checkmark & \text { Recuperación } \\ \checkmark & \text { Asimilación }\end{array}$

\section{Sesión de Activación o Entrenamiento}

Está conformado con las siguientes partes:

$\checkmark \quad$ Calentamiento: Consiste en una parte de trabajo de larga duración y baja intensidad, donde permita una buena tonificación muscular, seguidos de ejercicios de movilidad articular. 
$\checkmark \quad$ Ejercicios de Activación Específica: Remite ejecutar ejercicios propios del deporte que va a realizar, este son básicos de progresión, permite desarrollar elementos técnicos enfocados al desarrollo.

$\checkmark \quad$ Realizar Una Vuelta a la Calma Adecuada: En muchas de las ocasiones debemos realizar una carrera continua de baja intensidad de los ejercicios realizados.

\section{Componentes de la Carga de Entrenamiento}

Son los que provocan una adaptación a una sesión de entrenamiento es primordial considerar el proceso de estímulo y respuesta que provoca cambios en la estructura y función corporal. Los componentes de la carga pueden ser:

Intensidad:Marca el nivel de exigencia de trabajo a ejecutar.

Volumen: Cantidad de trabajo de forma absoluta o porcentual.

Densidad:Relación entre el esfuerzo y descanso.

Duración: Tiempo asignado al entrenamiento.

Frecuencia: Es la cantidad de número de sesiones por semana.

\section{Explicación de la Propuesta Escrita}

El presente Plan fue el inicio de un proceso de preparación en el mes de enero del 2015, con los estudiantes deportistas de baloncesto del circuito 06D01C02, el desarrollo de los fundamentos básicos técnicos del baloncesto con miras a campeonatos fue uno de los objetivos intrínsecos en la ejecución de las sesiones de entrenamiento.

Cabe indicar que el presente Plan del año 2015, tuvo una sola fase que culminó con los juegos intercolegiales tomando como competencia preparatoria los Juegos inter circuitales, además se aplicó en toda la Provincia como patrón para la preparación de los preseleccionados provinciales en cada uno de los circuitos que tuvieron basquetbolistas con proyecciones a mediano y largo plazo.

\section{Descripción de los Ciclos y Su Periodo de Duración}

1. Macrociclo: El Macro ciclo 2015 en su primera parte contiene un volumen total distribuido en repeticiones para los fundamentos técnicos, en kilómetros para la resistencia y en metros para la velocidad en los siguientes periodos:

1.1 Periodo Preparatorio: Que va desde lunes 5 de enero al 17 de abril de 2015, con un volumen parcial de 9.000 minutos, que equivale al $60 \%$ del Macro. Dividida en dos etapas: 
1.1.1 Etapa de Preparación Física General: Parte del 5 de Enero hasta el 6 de Abril del 2015, con un volumen parcial de 5.400, dando el 60\% del volumen del Periodo, contiene 7 Meso ciclos:

Introductorio, Desarrollador 1: Además tiene dos Controles Médicos tres Test Físico, y topes amistosos cinco.

1.1.2 Etapa de Preparación Física Especial: Parte desde el 9 de Marzo hasta el 17 de Abril del 2015, con un volumen parcial de 3.600 minutos, dando el $40 \%$ del volumen del Periodo, contiene dos Meso ciclos:

Desarrollador 2, Estabilizador 1: Además contiene Pruebas Médicas, un Test físicos y topes de preparación.

1.2 Periodo Competitivo: Va desde el 1 al 30 de Junio del 2015, con un volumen parcial de 3.000 minutos, dando un $35 \%$ del Macro. Dividida en dos Etapas:

1.3 Periodo de Transito: Va desde el 6 de al 31 de julio de 2015, con un volumen de 2400 minutos, dando un 5\% del volumen total del Macro, contiene un Meso ciclo recuperación, un Control Médico.

Plan de Desarrollo Físico - Técnico - Táctico- Teórico

2.1 Físicos Objetivos

Desarrollo de las capacidades físicas como: Resistencia, Fuerza, Velocidad.

\subsection{Técnicos Objetivos}

Consolidación de los Fundamentos Técnicos, como: Desplazamiento, Drible, Pases, Tiros, manejo de balón.

\subsection{Tácticos Objetivos}

Lograr la composición de los fundamentos básicos técnicos individual y grupal crear nuevas alternativas de juego para su mayor desempeño en la competencia.

\subsection{Teóricos Objetivos}

Lograr que el alumno conozca la simbología del baloncesto, acorde a los contenidos de la edad. Enseñar las reglas fundamentales del baloncesto, relacionadas con el juego y las competencias en correspondencia con las edades y ajustes de la FIBA.

\section{Objetivos de los Meso Ciclos}

\subsection{Mesociclo I Introductorio}

- $\quad$ Pruebas físicas iniciales

- Pruebas Médicas

\subsection{Mesociclo Desarrollador 1}

- Mejoramiento de las capacidades y habilidades motrices

- Bases desarrollar y ejecutar correctamente los fundamentos básicos técnicos del baloncesto 


\subsection{Mesociclo Desarrollador 2}

- Desarrollar el trabajo volitivo del equipo (Voluntad)

- Desarrollo De la capacidades físicas hasta un $70 \%$

\subsection{Mesociclo Estabilizador 1}

- Desarrollo de las cualidades físicas especiales

- Desarrollo de las cualidades técnico - tácticas

\subsection{Mesociclo Precompetitivo}

- Acondicionar al grupo para el juego

- Desarrollar nuestro sistema de juego

\subsection{Mesociclo Competitivo}

- Consolidar nuestro sistema defensivo para la competencia

- Consolidar nuestro sistema ofensivo para la competencia

\subsection{Mesociclo Tránsito}

- Recuperar mediante el descanso activo

- Pruebas Médicas

\section{Necesidades Para El Cumplimiento Del Plan}

$\checkmark 25$ Balones Para Entrenamientos

$\checkmark 12$ Balones Medicinales

$\checkmark$ Atención Médica Para Los Deportistas

$\checkmark$ Cumplimiento De Los Topes Amistosos

Se realizaron 29 sesiones en total correspondientes a los 7 mesociclos descritos, cada sesión forma parte del plan diario de entrenamiento distribuidos porcentualmente entre los 5 días de la semana y 7 meses respectivamente desde enero hasta julio del año 2015. A continuación se detallan siete sesiones que se realizaron con los estudiantes de la Unidad Educativa Riobamba.

\section{Unidad De Entrenamiento}

Provincia de Chimborazo

\section{Circuito 06D01C02 Distrito Chambo-Riobamba}

Plan Diario De Entrenamiento

\begin{tabular}{|c|c|c|c|c|}
\hline \multicolumn{3}{|c|}{ Lugar de la práctica deportiva: U.E "RIOBAMBA" } & & Deporte: Baloncesto \\
\hline \multicolumn{3}{|c|}{ Tiempo: 120 minutos } & \multicolumn{2}{|c|}{ Sesión: 1} \\
\hline \multirow[t]{2}{*}{ UNIDAD } & \multirow[t]{2}{*}{ ACTIVIDADES } & \multirow{2}{*}{$\begin{array}{c}\text { TIE } \\
\text { MP } \\
\text { O }\end{array}$} & $\begin{array}{l}\text { DOSIFIC } \\
\text { ACIÓN }\end{array}$ & \multirow[t]{2}{*}{$\begin{array}{l}\text { MEDIOS Y } \\
\text { MÉTODOS }\end{array}$} \\
\hline & & & $\begin{array}{l}\text { REPETIC } \\
\text { IONES }\end{array}$ & \\
\hline
\end{tabular}




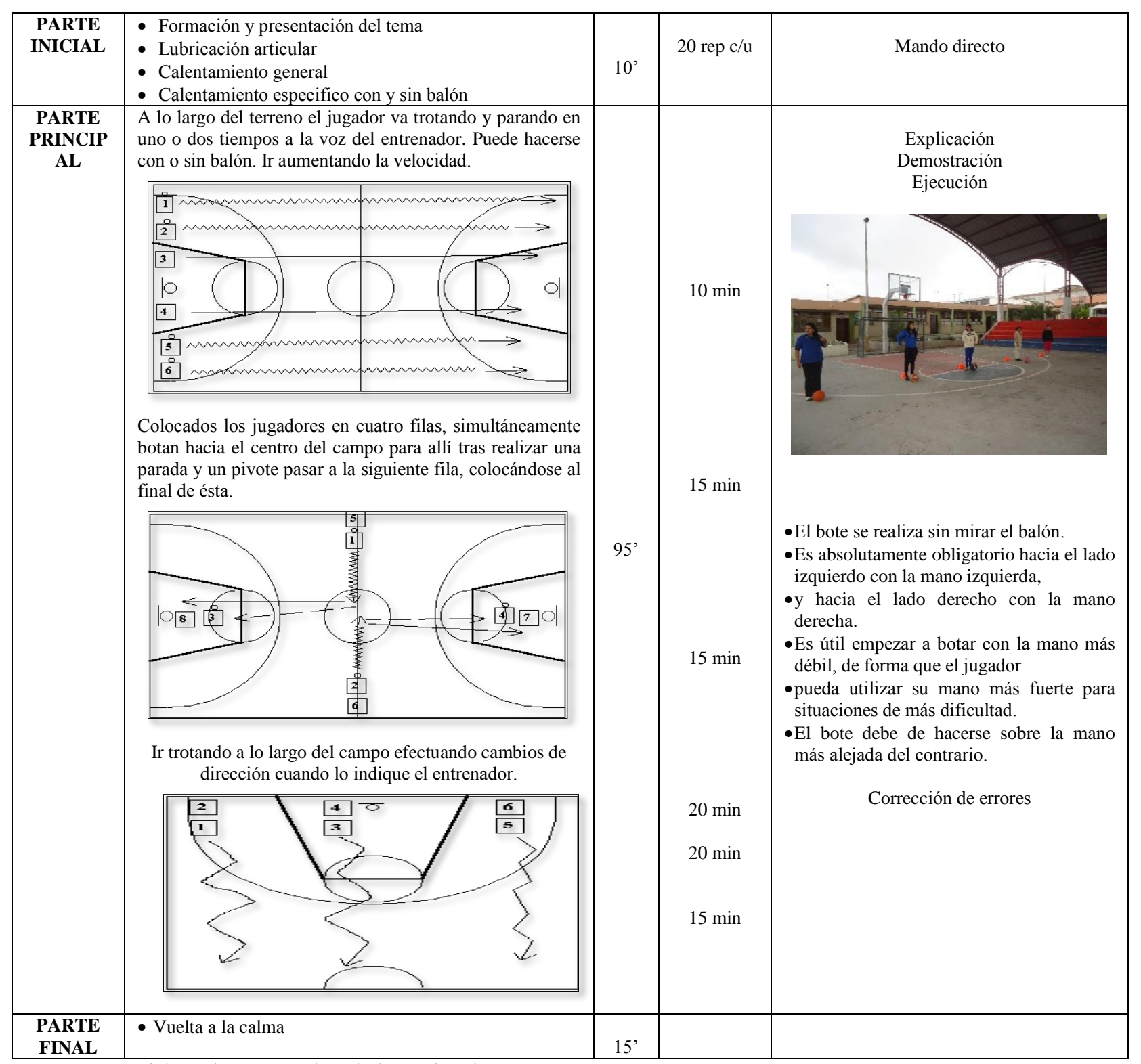

Elaborado por: equipo de investigación, 210 


\section{Unidad De Entrenamiento}

\section{Provincia de Chimborazo}

\section{Circuito 06D01C02 Distrito Chambo-Riobamba}

Plan Diario De Entrenamiento

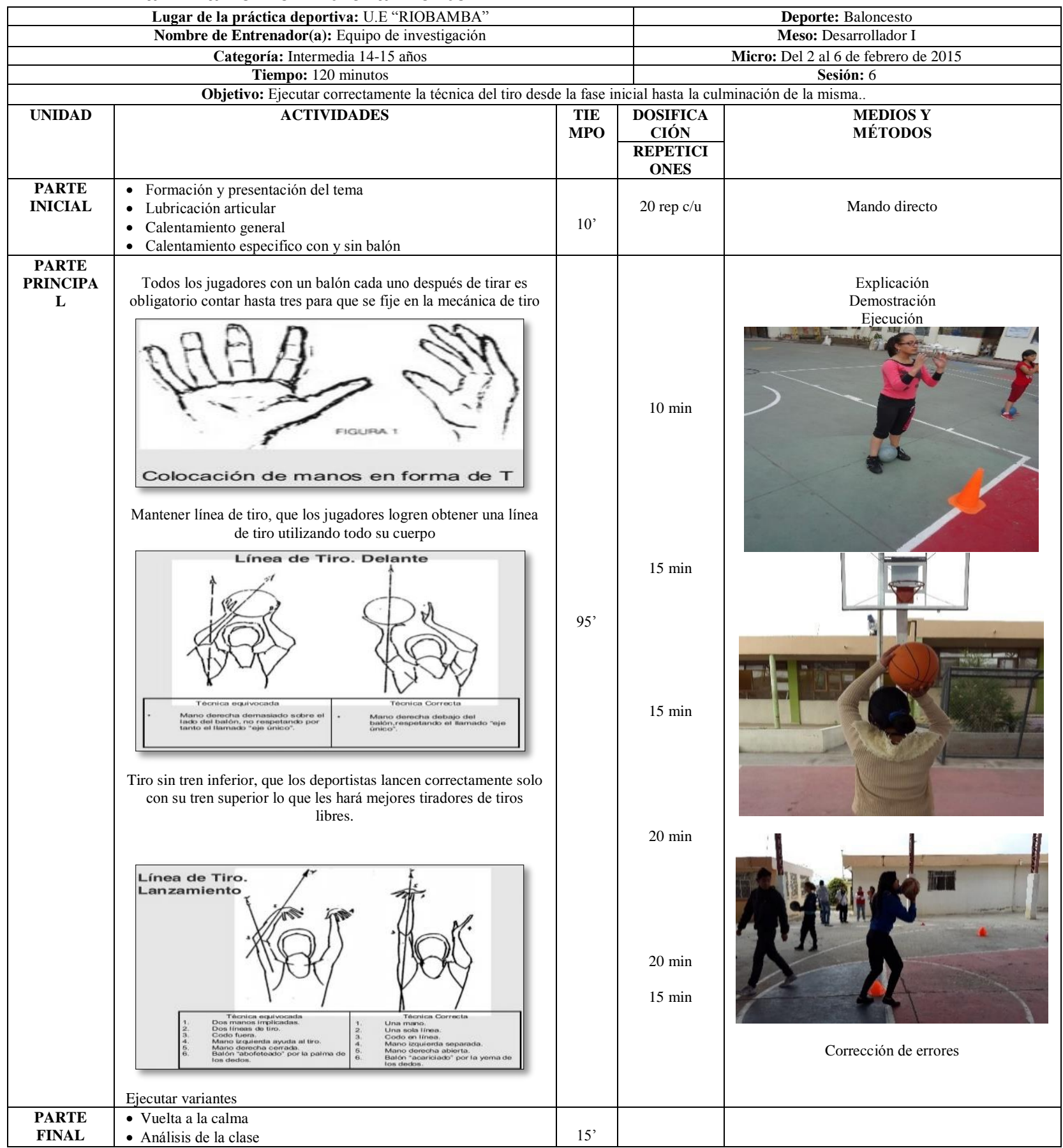

Elaborado por: equipo de investigación, 210 


\section{Unidad De Entrenamiento}

\section{Provincia de Chimborazo}

\section{Circuito 06D01C02 Distrito Chambo-Riobamba}

Plan Diario De Entrenamiento

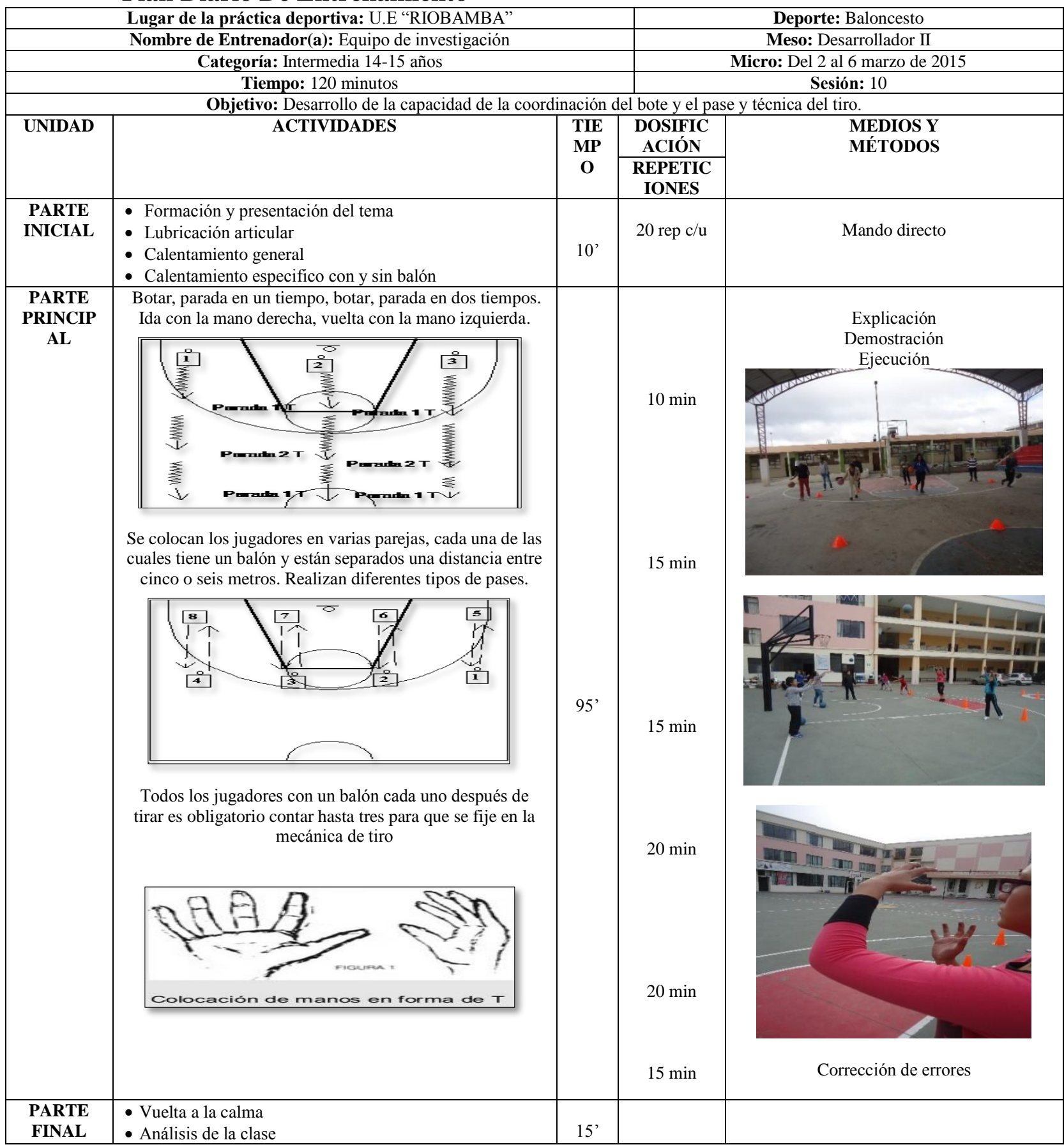

Elaborado por: equipo de investigación, 2105 


\section{Unidad De Entrenamiento}

\section{Provincia de Chimborazo}

\section{Circuito 06D01C02 Distrito Chambo-Riobamba}

\section{Plan Diario De Entrenamiento}

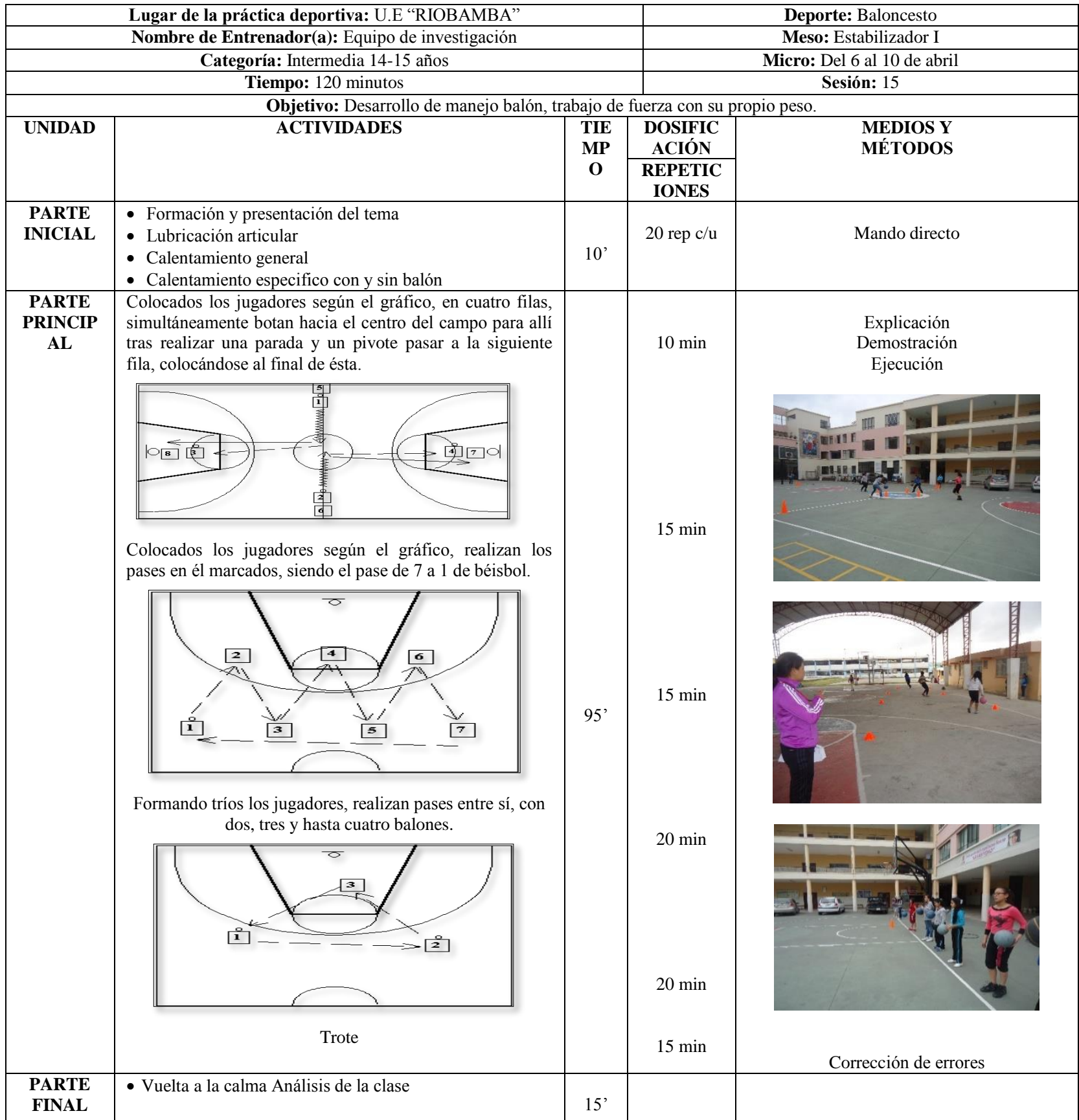

Elaborado por: equipo de investigación, 2105 


\section{Unidad De Entrenamiento}

\section{Provincia de Chimborazo}

\section{Circuito 06D01C02 Distrito Chambo-Riobamba}

Plan Diario De Entrenamiento

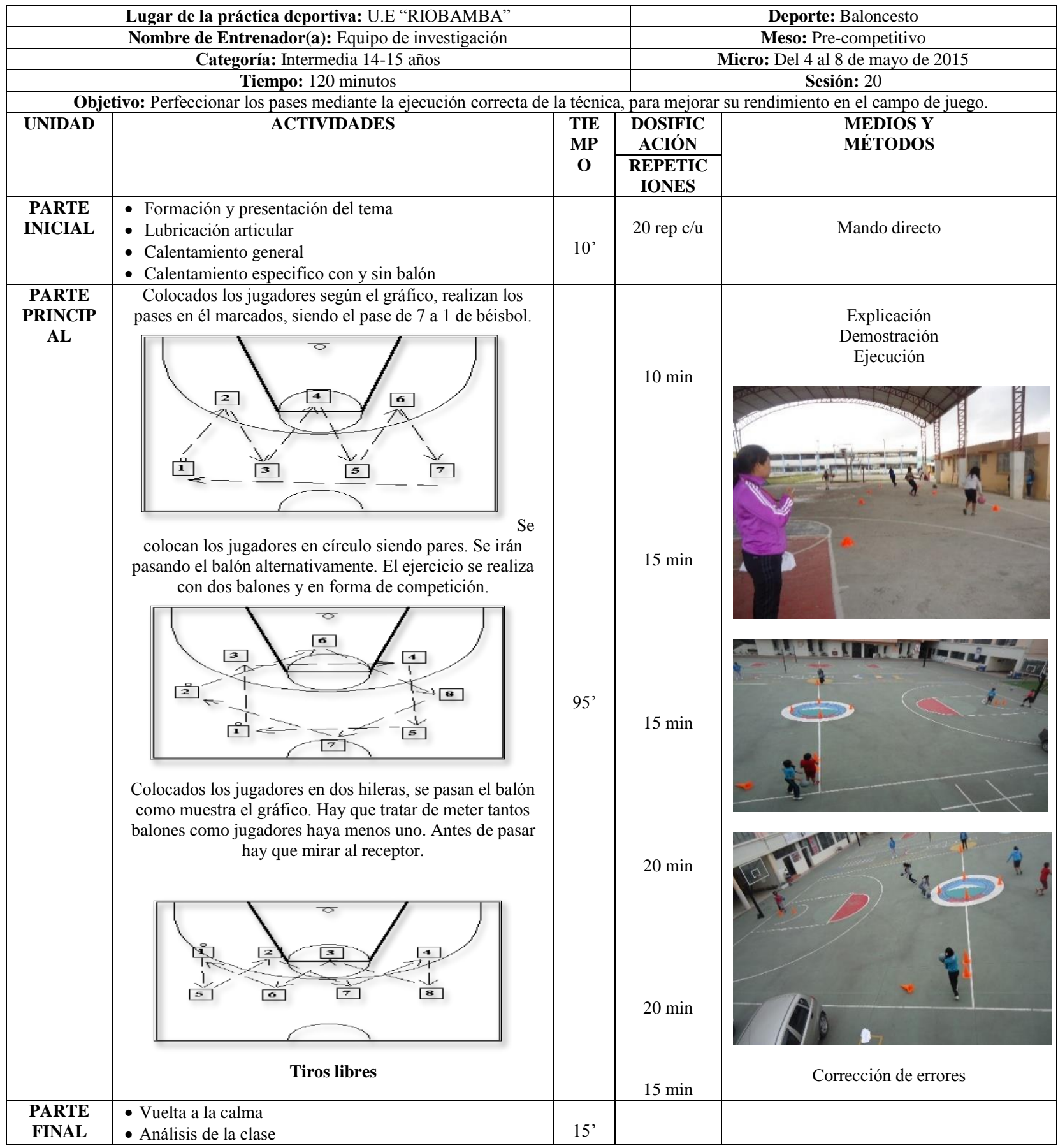

Elaborado por: equipo de investigación, 2105 


\section{Unidad De Entrenamiento}

\section{Provincia de Chimborazo}

\section{Circuito 06D01C02 Distrito Chambo-Riobamba}

Plan Diario De Entrenamiento

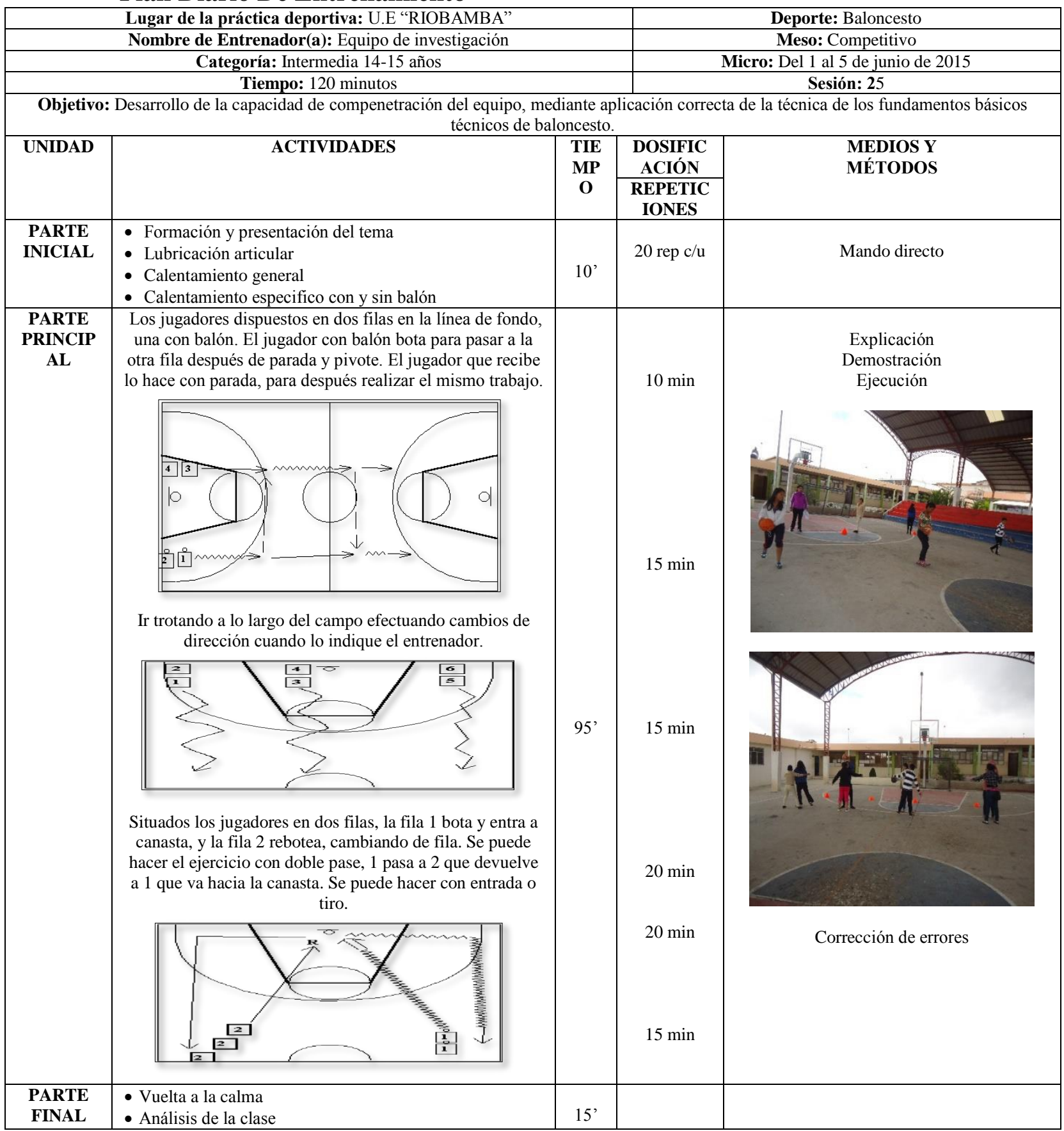

Elaborado por: equipo de investigación, 2105 


\section{Unidad De Entrenamiento}

\section{Provincia de Chimborazo}

\section{Circuito 06D01C02 Distrito Chambo-Riobamba}

Plan Diario De Entrenamiento

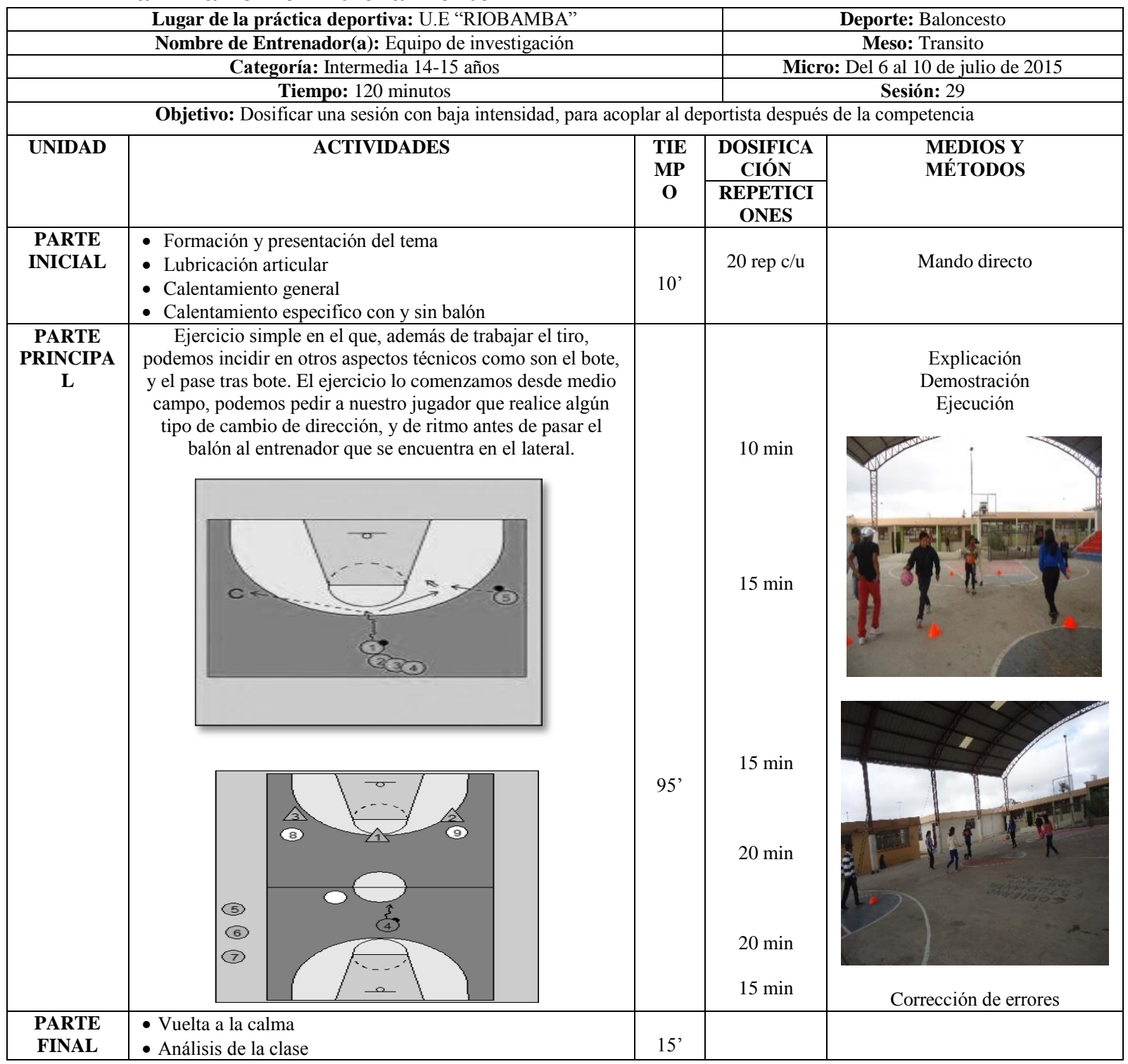

Elaborado por: equipo de investigación, 2105

\section{Conclusiones}

Se concluye que el estudio del "Análisis de los fundamentos técnicos del baloncesto" realizado a partir de las encuestas y observación a los estudiantes y entrenadores del circuito 06D01C02 del distrito Chambo Riobamba es un antecedente importante para elaborar el presente Plan de 
Entrenamiento para el Desarrollo de los Fundamentos Técnicos del Baloncesto, pues se refleja la problemática en el bajo rendimiento de los deportistas y la necesidad de crear un plan orientado a la mejora de esta situación de partida.

El uso del plan de entrenamiento ayuda a la reducción de gestos técnicos mal ejecutados, y es una herramienta que se adapta a las necesidades de los entrenadores y estudiantes.

Se determinó además que el rendimiento de los deportistas mejoró paulatinamente luego de ser aplicadas las 29 sesiones de entrenamiento, esto a través de los resultados expuestos en las competencias intercolegiales, en donde los estudiantes de las Unidades Educativas intervenidas mejoraron sus resultados en los distintos partidos.

\section{References:}

1. Alvarez, M. (2004). Planificación y periodización del entrenamiento en un equipo profesional de fútbol. Universidad de Málaga. Consultado el 20/08/2017. En línea. http://prof.webcindario.com/planificacion_periodizacion.pdf

2. Cando, K., Guitierrez, H., Ponce H., Aldas H. \& Mazón O. (2017). Análisis de los fundamentos técnicos del baloncesto en el rendimiento deportivo de los estudiantes categoría intermedia del circuito 06D01C02 distrito Chambo - Riobamba. European Scientific Journal. Vol 13.

3. Carrasco Dimas, Carrasco David \& Carrasco Darío (2014). Teoría y Práctica del Entrenamiento Deportivo. Universidad Politécnica de Madrid. Consultado el 20/08/2017. En línea. http://futbolcarrasco.com/wpcontent/uploads/2014/08/futbolcarrascoinef4curso9.pdf

4. Jiménez, J. (2011). Planificación del entrenamiento deportivo. Universidad de Antioquía. Primera edición. Funámbulos Editores. Medellín - Colombia. Consultado el 25/08/2017. En línea: http://viref.udea.edu.co/contenido/publicaciones/libros_expo2011/pla nificacion_entrenamiento_deportivo.pdf

5. Mestre, J. (2013). Componentes de la gestión deportiva, una aproximación. Revista de educación Física. Vol 2, Número 2. Universidad de Antioquía. Consultado el 24/08/2017. En línea. file:///C:/Users/user/Downloads/15775-53176-1-PB.pdf

6. Torrijo, J. (2004). Planificación y periodización del entrenamiento en un equipo base. Programa de Formación Permanente. Consultado el 25/08/2017.

En línea. http://www.cult.gva.es/dgd/form_amb_deportivo/JORNADA_FUTB OL_JUNIO/Futbol_Juan\%20Jose\%20Torrijo.pdf. 